\title{
Multisensor Switching Control Strategy with Fault Tolerance Guarantees
}

\author{
María M. Seron ${ }^{\mathrm{a}, 1}$ Xiang W. Zhuo a José A. De Doná ${ }^{\mathrm{a}}$ John J. Martínez ${ }^{\mathrm{b}}$ \\ ${ }^{a}$ Centre for Complex Dynamic Systems and Control (CDSC), School of Electrical Engineering and Computer Science, The \\ University of Newcastle, Callaghan, NSW 2308, Australia
}

${ }^{\mathrm{b}}$ Gipsa-lab UMR 5216, Département Automatique. BP.46, St. Martin D'Hères 38440, France

\begin{abstract}
In this paper we propose a novel fault tolerant multisensor switching strategy for feedback control. Each sensor of the proposed multisensor scheme has an associated state estimator which, together with a state feedback gain, is able to individually stabilise the closed loop system. At each instant of time, the switching strategy selects the sensor-estimator pair that provides the best closed loop performance, as measured by a control-performance criterion. We establish closed-loop stability of the resulting switching scheme under normal (fault-free) operating conditions. More importantly, we show that closed-loop stability is preserved in the presence of faulty sensors if a set of conditions on the system parameters (such as bounds on the sensor noises, maximum and minimum values of the reference signal, etc.) is satisfied. This result enhances and broadens the applicability of the proposed multisensor scheme since it provides guaranteed properties such as fault tolerance and robust closed-loop stability under sensor fault. The results are applied to the problem of automotive longitudinal control.
\end{abstract}

Key words: Multisensor systems, switching control, fault-tolerant systems, fault detection and isolation.

\section{Introduction}

Recent advances in sensor technology have generated substantial research interest in developing strategies for multisensor fusion, which aim at combining data supplied by different sensors to provide more accurate and reliable information. When compared with a system employing a single sensor, a multisensor system has enhanced properties such as improved reliability and robustness, extended coverage, increased confidence, faster responses and better resolution (Varshney, 1997). Numerous strategies for multisensor fusion have been proposed in the literature; see, e.g., Dasarathy (1997); Kalandros et al. (2004); Luo and Kay (1989); Luo et al. (2002); Sun and Deng (2004); Varshney (1997); Xu et al. (2004).

The use of sensor fusion estimates in feedback control systems has largely relied on ad-hoc techniques, whereby a multisensor fusion system and a controller are designed independently prior to their assembling within a feedback loop. Recent examples of this type of assembly

1 Corresponding author. Ph: +61 2 49216810, Fax: +61 2 49601712, email: Maria.Seron@newcastle.edu.au technique have been reported for automotive applications. For instance, Hsiao and Tomizuka (2005) combine a mixture Kalman filter having fault detection capabilities with an arbitrarily designed stabilising controller in a multisensor strategy for vehicle lateral control. The resulting scheme does not have pre-checkable fault tolerance guarantees but it performs well in simulations.

In this paper we introduce a novel switching approach for multisensor feedback control which has fault tolerance guarantees. Each sensor of the proposed multisensor scheme has an associated state estimator. At each instant of time, the switching strategy utilises a state feedback gain to feed back the estimate of the plant states that achieves the best value of a chosen control-performance criterion. We establish closed-loop stability of the resulting switching scheme under normal (fault-free) operating conditions. In particular, we show that, in the fault-free case, the system achieves asymptotic stability in the absence of disturbances and ultimate boundedness in the presence of bounded disturbances. A distinctive characteristic of the proposed scheme, when compared to sensor fusion approaches [for example, Hsiao and Tomizuka (2005); Sun and Deng (2004)], is that we allow for very general descriptions of the disturbances and do not require any particular prob- 
ability distribution, provided they are bounded. In addition, we provide conditions to guarantee closed-loop stability of the proposed switching scheme under the occurrence of abrupt faults in some of the sensors leading to sensor outage. These conditions are such that the switching scheme never selects failed sensors to implement the control law. These results enhance and broaden the applicability of the proposed multisensor scheme since they provide guaranteed properties such as fault tolerance and robust closed-loop stability under sensor fault.

The proposed switching scheme is applied to the problem of automotive vehicle longitudinal control. For this problem, we find conditions on the parameters of the system (such as bounds on the sensor noises, maximum and minimum values of the reference signal, etc.) to guarantee closed-loop stability under sensor fault. Simulation results under realistic assumptions confirm the validity of these conditions. In addition, a numerical example is presented to compare our proposed scheme with an alternative approach based on multisensor fusion.

\section{Multisensor switching scheme}

In this section, we describe the proposed multisensor switching control scheme, depicted in Figure 1.

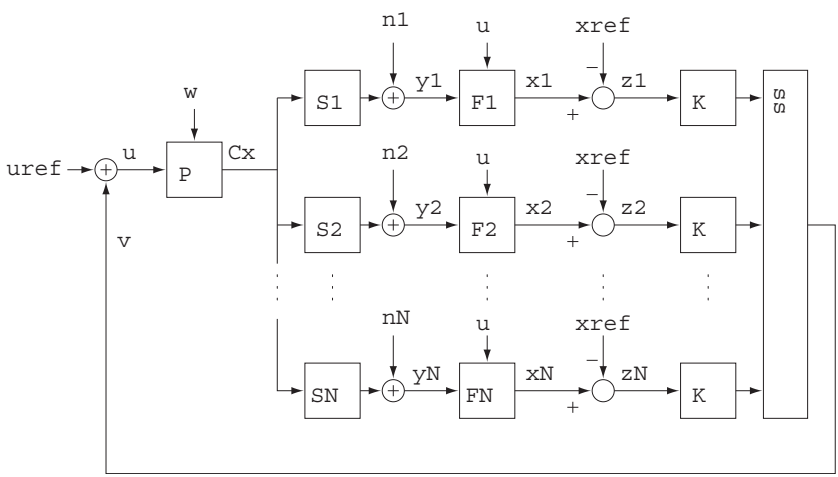

Fig. 1. Multisensor switching scheme with plant $P$, sensors $S_{1}, \ldots S_{N}$, estimators $F_{1}, \ldots, F_{N}$ and feedback gain $K$.

\subsection{Problem statement}

Consider the following linear discrete-time plant model

$$
x^{+}=A x+B u+E w,
$$

where $x \in \mathbb{R}^{n}$ and $x^{+} \in \mathbb{R}^{n}$ are, respectively, the current and successor system states, $u \in \mathbb{R}^{m}$ is the input, and $w \in \mathbb{R}^{r}$ is a bounded process disturbance.

Assumption 2.1 The pair $(A, B)$ is stabilisable.

$\circ$

The control objective is for the state of the plant (1) to track a reference signal $x_{r e f}$ that satisfies

$$
x_{r e f}^{+}=A x_{r e f}+B u_{r e f} .
$$

Assumption 2.2 The reference signals $u_{\text {ref }}$ and $x_{\text {ref }}$ in (2) are bounded.

Note that in the case where $A$ has eigenvalues on or outside the unit circle, $u_{\text {ref }}$ must be obtained from some stabilising feedback controller for system (2).

\subsection{Sensors, estimators, and tracking errors}

We assume that a plant's combination of states $C x \in \mathbb{R}^{p}$ is measured via a family of $N$ sensors

$$
\begin{aligned}
\xi_{i}^{+} & =A_{s_{i}} \xi_{i}+B_{s_{i}} C x \\
y_{i} & =C_{s_{i}} \xi_{i}+\eta_{i}, \quad i=1, \cdots, N,
\end{aligned}
$$

where, for each sensor, $\xi_{i} \in \mathbb{R}^{n_{i}}$ is the state, $y_{i} \in \mathbb{R}^{p_{i}}$ is the measured output and $\eta_{i} \in \mathbb{R}^{p_{i}}$ is a bounded measurement disturbance.

Assumption 2.3 The matrices $A_{s_{i}}$ in (3) have all their eigenvalues strictly inside the unit circle.

Assumption 2.4 The pairs $\left(\left[\begin{array}{cc}A & 0 \\ B_{s_{i}} C & A_{s_{i}}\end{array}\right],\left[\begin{array}{ll}0 & C_{s_{i}}\end{array}\right]\right)$ are detectable for $i=1, \ldots, N$.

For future reference, we define sensor "reference signals" $\xi_{i, r e f}, i=1, \ldots, N$, as the solution to

$$
\xi_{i, r e f}^{+}=A_{s_{i}} \xi_{i, r e f}+B_{s_{i}} C x_{r e f} .
$$

Note that $\xi_{i, r e f}$ are bounded by Assumptions 2.2 and 2.3.

We consider $N$ state estimators, each of which estimates the states of the series connection of the plant and a sensor. The estimators are described by the following dynamic and update equations, for $i=1, \cdots, N$ :

$$
\begin{aligned}
\hat{x}_{i}^{+} & =A \hat{x}_{i}+B u+L_{i}\left(y_{i}-C_{s_{i}} \hat{\xi}_{i}\right), \\
\hat{\xi}_{i}^{+} & =A_{s_{i}} \hat{\xi}_{i}+B_{s_{i}} C \hat{x}_{i}+L_{s_{i}}\left(y_{i}-C_{s_{i}} \hat{\xi}_{i}\right), \\
\hat{x}_{i}^{U P} & =\hat{x}_{i}+M_{i}\left(y_{i}-C_{s_{i}} \hat{\xi}_{i}\right), \\
\hat{\xi}_{i}^{U P} & =\hat{\xi}_{i}+M_{s_{i}}\left(y_{i}-C_{s_{i}} \hat{\xi}_{i}\right) .
\end{aligned}
$$

Assumption 2.5 The gains $L_{i}, L_{s_{i}}$ are such that

$$
A_{L_{i}} \triangleq\left[\begin{array}{cc}
A & 0 \\
B_{s_{i}} C & A_{s_{i}}
\end{array}\right]-\left[\begin{array}{c}
L_{i} \\
L_{s_{i}}
\end{array}\right]\left[\begin{array}{ll}
0 & C_{s_{i}}
\end{array}\right]
$$

for $i=1, \cdots, N$, have all their eigenvalues strictly inside the unit circle [note that this is always possible by 
Assumption 2.4]. The update gains $M_{i}$ and $M_{s_{i}}$ are arbitrary real matrices of appropriate dimensions. ${ }^{2}$

Remark 2.6 The estimation errors, defined as

$$
\left[\begin{array}{c}
\tilde{x}_{i} \\
\tilde{\xi}_{i}
\end{array}\right] \triangleq\left[\begin{array}{c}
x-\hat{x}_{i} \\
\xi_{i}-\hat{\xi}_{i}
\end{array}\right], \quad i=1, \cdots, N
$$

satisfy, using (1), (3), (4), (6), (7) and (10),

$$
\left[\begin{array}{c}
\tilde{x}_{i}^{+} \\
\tilde{\xi}_{i}^{+}
\end{array}\right]=A_{L_{i}}\left[\begin{array}{c}
\tilde{x}_{i} \\
\tilde{\xi}_{i}
\end{array}\right]+\left[\begin{array}{c}
E \\
0
\end{array}\right] w-\left[\begin{array}{c}
L_{i} \\
L_{s_{i}}
\end{array}\right] \eta_{i} .
$$

Hence, it follows from Assumption 2.5 that $\tilde{x}_{i}$ and $\tilde{\xi}_{i}$ are bounded whenever $w$ and $\eta_{i}$ are bounded.

We will study the plant tracking error defined as

$$
z \triangleq x-x_{r e f}
$$

In addition, we define "tracking errors" for the input, sensor states, plant and sensor state estimates, and plant state updated estimates, respectively, as

$$
\begin{aligned}
v & \triangleq u-u_{\text {ref }}, \\
\zeta_{i} & \triangleq \xi_{i}-\xi_{i, r e f}, \\
\hat{z}_{i} & \triangleq \hat{x}_{i}-x_{\text {ref }}, \\
\hat{\zeta}_{i} & \triangleq \hat{\xi}_{i}-\xi_{i, r e f}, \\
\hat{z}_{i}^{U P} & \triangleq \hat{x}_{i}^{U P}-x_{r e f}=\hat{z}_{i}+\gamma_{i},
\end{aligned}
$$

for $i=1, \cdots, N$, where

$$
\gamma_{i} \triangleq M_{i}\left(y_{i}-C_{s_{i}} \hat{\xi}_{i}\right)
$$

Substituting (4) and (11) in (19), we have

$$
\gamma_{i}=M_{i} C_{s_{i}} \tilde{\xi}_{i}+M_{i} \eta_{i}
$$

\subsection{Switching controller}

We propose a switching controller that at each time instant selects the sensor-estimator pair, and computes the corresponding feedback control action, so that the following switching cost function is minimised:

$$
J(\hat{z}, v)=\hat{z}^{\prime} Q \hat{z}+v^{\prime} R v+(A \hat{z}+B v)^{\prime} P(A \hat{z}+B v),
$$

2 If the estimators are steady-state Kalman filters then $L_{i}$, $L_{s_{i}}, M_{i}$ and $M_{s_{i}}$ are obtained via an algebraic Riccati equation. More generally, $L_{i}, L_{s_{i}}$ can be computed by placement of the poles of $A_{L_{i}}$ in some desired location. where $\hat{z} \in \hat{Z}^{U P} \triangleq\left\{\hat{z}_{1}^{U P}, \cdots, \hat{z}_{N}^{U P}\right\}$ and $\hat{z}_{i}^{U P}, i=$ $1, \ldots, N$, are the tracking errors for the updated estimates of the plant states, defined in (18). In (21), $Q>0$ and $R>0$ are design (tuning) parameters, and $P>0$ is computed from the algebraic Riccati equation [ARE]

$$
\begin{aligned}
& P=A^{\prime} P A+Q-K^{\prime}\left(R+B^{\prime} P B\right) K, \\
& K \triangleq\left(R+B^{\prime} P B\right)^{-1} B^{\prime} P A .
\end{aligned}
$$

At each time step, we perform the optimisation

$$
\left(\hat{z}^{*}, v^{*}\right)=\arg \min _{(\hat{z}, v)}\left\{J(\hat{z}, v): \hat{z} \in \hat{Z}^{U P}, v \in \mathbb{R}^{m}\right\},
$$

and the control is computed from (14) as $u^{*}=u_{\text {ref }}+v^{*}$.

From the choices (22) in the cost function, it follows that, for a fixed $\hat{z}$, the minimisation of (21) with respect to $v$ is equivalent to the standard steadystate linear quadratic control problem of minimising $\sum_{k=0}^{\infty}\left[\hat{z}(k)^{\prime} Q \hat{z}(k)+v(k)^{\prime} R v(k)\right]$ subject to the dynamic equation $\hat{z}(k+1)=A \hat{z}(k)+B v(k), \hat{z}(0)=\hat{z}$.

Hence $v=-K \hat{z}$, with $K$ defined in (22), minimises (21) with respect to $v$, that is, $\min _{v}\{J(\hat{z}, v): v \in \mathbb{R}\}=$ $J(\hat{z},-K \hat{z})=\hat{z}^{\prime} Q \hat{z}+\hat{z}^{\prime} K^{\prime} R K \hat{z}+\hat{z}^{\prime}(A-B K)^{\prime} P(A-$ $B K) \hat{z}$. Using (22) we have $Q+K^{\prime} R K+(A-B K)^{\prime} P(A-$ $B K)=P$, and hence $\min _{v}\{J(\hat{z}, v): v \in \mathbb{R}\}=\hat{z}^{\prime} P \hat{z}$. It follows that $v^{*}=-K \hat{z}^{*}$ in (23) and, correspondingly,

$$
u^{*}=u_{\text {ref }}-K \hat{z}^{*}, \hat{z}^{*}=\arg \min _{\hat{z}}\left\{\hat{z}^{\prime} P \hat{z}: \hat{z} \in \hat{Z}^{U P}\right\},
$$

and the minimum is given by

$$
J\left(\hat{z}^{*},-K \hat{z}^{*}\right)=\left(\hat{z}^{*}\right)^{\prime} P \hat{z}^{*}
$$

Thus, at each time instant, the switching controller feeds back the best, with respect to the minimisation (23), updated estimate tracking error via a linear gain $K$. Matrix $P$ in (25) is the solution of the ARE and $K$ is the associated linear state feedback gain. In particular, Assumption 2.1 guarantees that $K$ is such that $A-B K$ has all its eigenvalues strictly inside the unit circle.

\section{Closed-loop stability and ultimate bounds in the absence of sensor fault}

In this section we first establish closed-loop stability of the switching scheme described in Section 2 under full operability of all sensors, that is, in the absence of sensor fault. We then derive ultimate bounds on the closedloop system's states that will hold if the system has been running without sensor fault for sufficiently long time. These bounds will be used in Section 4 when we obtain conditions for stability under sensor fault. 


\subsection{Closed-loop stability}

Theorem 3.1 Under Assumptions 2.1 to 2.5, the switching control system described in Section 2 has bounded trajectories. Moreover, the plant tracking error (13) and the estimator tracking errors (16), for $i=1, \ldots, N$, asymptotically converge to zero in the absence of process and measurement disturbances.

PROOF. Let system (1), (3)-(4) and (6)-(9) be in closed-loop with control $u=u^{*}=u_{\text {ref }}-K \hat{z}^{*}$ given by (24). Suppose that, at any arbitrary time, the switching controller selects some $l \in\{1, \cdots, N\}$ so that $\hat{z}^{*}=\hat{z}_{l}^{U P}$, $u^{*}=u_{r e f}-K \hat{z}_{l}^{U P}$. Using (18) and (16), we have

$$
u^{*}=u_{r e f}-K\left(\hat{z}_{l}+\gamma_{l}\right)=u_{r e f}-K\left(\hat{x}_{l}-x_{r e f}+\gamma_{l}\right)
$$

Substituting (26) into (1) and using (13), (2), (11) and (20), we have that the plant tracking error satisfies

$$
\begin{aligned}
& z^{+}=x^{+}-x_{r e f}^{+}=(A-B K) z+ \\
& {\left[E B K-B K M_{l} C_{s_{l}}-B K M_{l}\right]\left[\begin{array}{llll}
w^{\prime} & \tilde{x}_{l}^{\prime} & \tilde{\xi}_{l}^{\prime} & \eta_{l}^{\prime}
\end{array}\right]^{\prime} .}
\end{aligned}
$$

In addition, from (16), (11) and (13), we have that, for all $i=1, \ldots, N$, the estimator tracking errors satisfy $\hat{z}_{i}=z-\tilde{x}_{i}$. Since $A-B K$ has all its eigenvalues strictly inside the unit circle and $w, \tilde{x}_{i}, \tilde{\xi}_{i}$ and $\eta_{i}$ are bounded for all $i \in\{1, \ldots, N\}$ (see Remark 2.6) it then follows that the tracking error $z$ and the estimator tracking errors $\hat{z}_{i}$, $i=1, \ldots, N$, are bounded. Moreover, they asymptotically converge to zero in the absence of disturbances. Using (3), (13), (15) and (5) we have the following sensor tracking error dynamics, for $i=1, \ldots, N$ :

$$
\zeta_{i}^{+}=A_{s_{i}} \zeta_{i}+B_{s_{i}} C z
$$

By Assumption 2.3 and since $z$ is bounded (as shown above), it follows from (28) that the sensor tracking errors are bounded for all $i=1, \ldots, N$. A similar analysis using (7) and (17) shows that the tracking errors $\hat{\zeta}_{i}$ for the sensor state estimates are bounded for all $i=1, \ldots, N$. The result then follows.

Theorem 3.1 is important since closed-loop stability is not automatically guaranteed for switched systems even if the controller switches between stable systems. Indeed, one can construct examples of switching between stable systems and a switching sequence that sends all trajectories to infinity [see, for example, Branicky (1998)].

\subsection{Ultimate bounds}

We present below a theorem that will allow us to compute ultimate bounds for the closed-loop system's states.
This result, whose proof can be consulted in Seron et al. (2006), extends Theorem 4.1 of Kofman et al. (2007) to a class of switched systems with constant perturbation bounds. In the sequel, $|M|$ denotes the elementwise magnitude of a (possibly complex) matrix $M$ and $x \leq y(x<y)$ denotes the set of elementwise (strict) inequalities between the components of the real vectors $x$ and $y$, and similarly for $x \geq y(x>y)$. In addition, if $W_{l}=\left[w_{l}^{1} \ldots w_{l}^{n}\right]^{\prime}, l \in\{1, \ldots, N\}$, are vectors in $\mathbb{R}^{n}$ then $\max _{l \in\{1, \ldots, N\}} W_{l}$ denotes the elementwise maximum, whose $i$ th element, $i=1, \ldots, n$, is defined as

$$
\left(\max _{l \in\{1, \ldots, N\}} W_{l}\right)_{i} \triangleq \max \left\{w_{1}^{i}, \ldots w_{N}^{i}\right\}
$$

Theorem 3.2 Consider the system $x(k+1)=$ $A x(k)+B_{l} \nu_{l}(k)$, where $A \in \mathbb{R}^{n \times n}, B_{l} \in \mathbb{R}^{n \times m}$, $l \in\{1, \ldots, N\}$, and $A$ has eigenvalues strictly inside the unit circle. Let $V \Lambda V^{-1}$ be the Jordan matrix decomposition of $A$. Assume that, for all $l \in\{1, \ldots, N\}$, $\left|\nu_{l}(k)\right| \leq \bar{\nu}_{l}$ for all $k \geq 0$, where $\bar{\nu}_{l} \in \mathbb{R}^{m}, \bar{\nu}_{l}>0$, and let $\bar{\nu} \triangleq \max _{l \in\{1, \ldots, N\}}\left|V^{-1} B_{l}\right| \bar{\nu}_{l}$. For $\epsilon \in \mathbb{R}^{n}, \epsilon \geq 0$, define

$$
S_{\epsilon} \triangleq\left\{x \in \mathbb{R}^{n}:\left|V^{-1} x\right| \leq(I-|\Lambda|)^{-1} \bar{\nu}+\epsilon\right\}
$$

Then:

(1) For any $\epsilon \geq 0$, the set $S_{\epsilon}$ is (positively) invariant. That is, if $\bar{x}(0) \in S_{\epsilon}$, then $x(k) \in S_{\epsilon}$ for all $k \geq 0$.

(2) Given $\epsilon \in \mathbb{R}^{n}, \epsilon>0$, there exists $k^{*} \geq 0$ such that $x(k) \in S_{\epsilon}$ for all $k \geq k^{*}$.

Remark 3.3 Part 1 of Theorem 3.2 characterises invariant sets in the state space, the smallest being the set $S_{0}$ obtained by taking $\epsilon=0$ in (30). Part 2 shows that the state trajectories asymptotically converge to the invariant set $S_{\epsilon}$ for $\epsilon \geq 0$ (in particular, $S_{0}$ ) from any initial condition. In addition, for $\epsilon>0$, the state trajectories enter $S_{\epsilon}$ in finite time. Note that an elementwise ultimate bound on the state can be obtained from Theorem 3.2 using the fact that $|x(k)| \leq|V|\left|V^{-1} x(k)\right|$.

Remark 3.4 If the eigenvalues of $A=V \Lambda V^{-1}$ are real, then the sets $S_{\epsilon}$ in (30) are polyhedral sets.

Remark 3.5 Assume that bounds on the measurement disturbances $\left|\eta_{i}\right| \leq \bar{\eta}_{i}$, for $i=1, \ldots, N$, and process disturbance $|w| \leq \bar{w}$ are problem data. ${ }^{3}$ Applying Theorem 3.2 to the estimation error subsystems (12), we obtain the following invariant sets in which each subsystem's trajectories will remain if started inside or towards which the trajectories will asymptotically converge

3 In the sequel, if $\nu(k) \in \mathbb{R}^{m}$ is a discrete-time signal and $\bar{\nu} \in \mathbb{R}^{m}, \bar{\nu} \geq 0$, then $|\nu| \leq \bar{\nu}$ denotes the elementwise bound $|\nu(k)| \leq \bar{\nu}$ for all times $k \geq k^{*}$, for some $k^{*} \geq 0$. 
if started outside:

$$
\begin{aligned}
\tilde{S}_{i} \triangleq\{ & {\left[\begin{array}{c}
\tilde{x}_{i} \\
\tilde{\xi}_{i}
\end{array}\right] \in \mathbb{R}^{n+n_{i}}:\left|V_{i}^{-1}\left[\begin{array}{c}
\tilde{x}_{i} \\
\tilde{\xi}_{i}
\end{array}\right]\right| \leq } \\
& \left.\left(I-\left|\Lambda_{i}\right|\right)^{-1}\left|V_{i}^{-1}\left[\begin{array}{cc}
E & -L_{i} \\
0 & -L_{s_{i}}
\end{array}\right]\right|\left[\begin{array}{c}
\bar{w} \\
\bar{\eta}_{i}
\end{array}\right]\right\},
\end{aligned}
$$

for $i=1, \ldots, N$, where $A_{L_{i}}=V_{i} \Lambda_{i} V_{i}^{-1}$ is the Jordan decomposition of $A_{L_{i}}$. From (31), we can compute ultimate bounds on $\tilde{x}_{i}$ and $\tilde{\xi}_{i}$, as suggested in Remark 3.3:

$$
\left|\begin{array}{c}
\tilde{x}_{i} \\
\tilde{\xi}_{i}
\end{array}\right| \leq\left|V_{i}\right|\left(I-\left|\Lambda_{i}\right|\right)^{-1}\left|V_{i}^{-1}\left[\begin{array}{cc}
E & -L_{i} \\
0 & -L_{s_{i}}
\end{array}\right]\right|\left[\begin{array}{c}
\bar{w} \\
\bar{\eta}_{i}
\end{array}\right] .
$$

Then, from (27), (28) and the bounds (32), we can obtain ultimate bounds on $z$, and then on $\zeta_{i}$, again using Theorem 3.2 and Remark 3.3.

\subsection{Closed-loop dynamics in the absence of fault}

We will consider the dynamics of the estimator tracking errors $\hat{z}_{i}$ and $\hat{\zeta}_{i}$ in closed loop with (26). Using equations from Sections 2.1 and 3.1 and the definitions

$$
\begin{aligned}
B_{l i} & \triangleq\left[\begin{array}{cccccc}
-B K & B K & -B K M_{l} C_{s_{l}} & -B K M_{l} & L_{i} C_{s_{i}} & L_{i} \\
0 & 0 & 0 & 0 & L_{s_{i}} C_{s_{i}} & L_{s_{i}}
\end{array}\right], \\
\nu_{l i} & \triangleq\left[\begin{array}{lllllll}
z^{\prime} & \tilde{x}_{l}^{\prime} & \tilde{\xi}_{l}^{\prime} & \eta_{l}^{\prime} & \zeta_{i}^{\prime} & \eta_{i}^{\prime}
\end{array}\right]^{\prime},
\end{aligned}
$$

we obtain the following closed-loop estimator tracking error subsystems (see Seron et al. (2006) for details):

$$
\left[\begin{array}{c}
\hat{z}_{i}^{+} \\
\hat{\zeta}_{i}^{+}
\end{array}\right]=A_{L_{i}}\left[\begin{array}{c}
\hat{z}_{i} \\
\hat{\zeta}_{i}
\end{array}\right]+B_{l i} \nu_{l i}, \quad i=1, \ldots, N,
$$

and $l$ varying in $\{1, \ldots, N\}$ [recall that $l$ represents the updated estimate tracking error $\hat{z}_{l}^{U P}$ selected at a given time instant in (26)]. Combining bounds on $z, \tilde{x}_{i}, \tilde{\xi}_{i}$ and $\zeta_{i}$, for $i=1, \ldots, N$, computed as discussed in Remark 3.5, readily yields a bound $\bar{\nu}_{l i}$ such that $\left|\nu_{l i}\right| \leq \bar{\nu}_{l i}$. Using (30) (with $\epsilon=0$ ) we can then compute the set

$$
\begin{aligned}
\hat{S}_{i} \triangleq & \left\{\left[\begin{array}{c}
\hat{z}_{i} \\
\hat{\zeta}_{i}
\end{array}\right] \in \mathbb{R}^{n+n_{i}}:\left|V_{i}^{-1}\left[\begin{array}{c}
\hat{z}_{i} \\
\hat{\zeta}_{i}
\end{array}\right]\right| \leq\right. \\
& \left.\left(I-\left|\Lambda_{i}\right|\right)^{-1} \max _{l \in\{1, \ldots, N\}}\left|V_{i}^{-1} B_{l i}\right| \bar{\nu}_{l i}\right\},
\end{aligned}
$$

where the maximum is taken elementwise [cf. (29)]. Thus, it follows from Theorem 3.2 that, in the absence of sensor fault, the trajectories of (34) will remain in $\hat{S}_{i}$ if started inside or will asymptotically converge towards $\hat{S}_{i}$ if started outside.

\section{Closed-loop stability under sensor fault}

Our fault model is described in the following definition. We consider abrupt faults that lead to sensor outage.

Definition 1 A sensor is operational (or "healthy") when its measured output is given by (4). When a jth sensor fails, its dynamics continue to obey (3), while its measured output during the fault is given by

$$
y_{j}=\eta_{j}^{F},
$$

where $\eta_{j}^{F}$ is a bounded noise.

In the following subsections we shall establish closedloop stability under sensor fault by providing conditions that guarantee that the switching scheme never selects faulty sensors to implement the control law.

\subsection{Closed-loop dynamics during the fault}

In this section we analyse the behaviour of the closedloop system under the following working hypothesis:

Assumption 4.1 The switching scheme always selects only healthy sensors whose estimation errors satisfy (32).

Section 4.2 will then derive conditions that ensure that our working hypothesis is satisfied.

\subsubsection{Healthy sensors}

Provided only healthy sensors are selected by the switching controller, the closed-loop dynamics of the estimator tracking errors for each of the $i$ th sensors that remain healthy continue to obey (34), that is, do not change in the event a $j$ th sensor fails. Moreover, the bounds that define the sets $\hat{S}_{i}$, namely $\left|\nu_{l i}\right| \leq \bar{\nu}_{l i}$, remain valid while (32) holds for the selected sensor [see (35) and (33)]. Thus, under Assumption 4.1, if the trajectories of healthy sensors (34) are evolving in the corresponding set $\hat{S}_{i}$, then they remain in this set.

\subsubsection{Faulty sensors}

Assuming that the switching scheme only selects healthy sensors $l \in\{1, \cdots, N\}, l \neq j$, then using equations from Sections 2.1 and 3.1 together with (36), $B_{l i}$ in (33) and

$$
\nu_{l j}^{F} \triangleq\left[z^{\prime} \tilde{x}_{l}^{\prime} \tilde{\xi}_{l}^{\prime} \eta_{l}^{\prime}-\xi_{j, r e f}^{\prime} \eta_{j}^{F^{\prime}}\right]^{\prime},
$$

we have the following closed-loop estimator tracking error subsystems during the fault:

$$
\left[\begin{array}{c}
\hat{z}_{j}^{+} \\
\hat{\zeta}_{j}^{+}
\end{array}\right]=A_{L_{j}}\left[\begin{array}{c}
\hat{z}_{j} \\
\hat{\zeta}_{j}
\end{array}\right]+B_{l j} \nu_{l j}^{F}, \quad j=1, \ldots, N,
$$


and $l$ varying in $\{1, \ldots, N\}, l \neq j$. Comparing (38)-(37) with (34)-(33), we observe that some of the inputs to the tracking error subsystems have changed after the fault. However, under Assumption 4.1, the signals $z, \tilde{x}_{l}, \tilde{\xi}_{l}$, for all operational sensors $l \in\{1, \ldots, N\}, l \neq j$, satisfy the same bounds as before the fault. In addition, $\xi_{j, r e f}$ and $\eta_{j}^{F}$, for $j=1, \ldots, N$, are bounded by assumption (we can readily obtain bounds on $\xi_{j, r e f}$ from (5) since, in practice, bounds on the reference signal $x_{r e f}$ are known). Hence, as before, we can use these different bounds to obtain a bound $\bar{\nu}_{l j}^{F}$ such that $\left|\nu_{l j}^{F}\right| \leq \bar{\nu}_{l j}^{F}$. Using (30) (with $\epsilon=0)$ we can then compute the "under-fault" set

$$
\begin{aligned}
\hat{S}_{j}^{F} \triangleq\{ & {\left[\begin{array}{l}
\hat{z}_{j} \\
\hat{\zeta}_{j}
\end{array}\right] \in \mathbb{R}^{n+n_{j}}:\left|V_{j}^{-1}\left[\begin{array}{c}
\hat{z}_{j} \\
\hat{\zeta}_{j}
\end{array}\right]\right| \leq } \\
& \left.\left(I-\left|\Lambda_{j}\right|\right)^{-1} \max _{l \in\{1, \ldots, N\}}\left|V_{j}^{-1} B_{l j}\right| \bar{\nu}_{l j}^{F}\right\},
\end{aligned}
$$

where the maximum is taken elementwise [cf. (29)]. Thus, it follows from Theorem 3.2 and the previous analysis that, under Assumption 4.1, the trajectories of (38) remain in $\hat{S}_{j}^{F}$ defined in (39) if started inside or will asymptotically converge towards $\hat{S}_{j}^{F}$ if started outside.

We are now ready to close the circle of ideas by making provisions to ensure that our working hypothesis is satisfied. This will be performed in two steps in the following subsection. The first step is to specify a fault scenario which describes the kind of fault situations that the proposed framework allows. The second step involves deriving conditions which ensure that, while the trajectories associated with healthy sensors are in the sets (35) and those associated with faulty sensors are in the sets (39), the optimisation (21)-(23) used by the switching scheme always favours a healthy sensor over any faulty sensor.

\subsection{Conditions for closed-loop stability}

The analysis of Section 4.1 motivates us to impose the following assumption, which describes the less conservative fault scenario that allows us to obtain fault tolerance guarantees within the proposed framework.

\section{Assumption 4.2 (Fault scenario)}

(1) At any time instant, at least one of the following two situations is true:

(a) all sensors are operational;

(b) at least one sensor is operational; in addition, all operational sensors have estimation errors inside the invariant sets $\tilde{S}_{i}(31)$, and at least one operational lth sensor has the states of the corresponding estimator tracking error subsystem (34) in the invariant set $\hat{S}_{l}(35)$.
(2) Any time a jth sensor fails, for any $j \in\{1, \ldots, N\}$, the states of the corresponding estimator tracking error subsystem (38) belong to the invariant set $\hat{S}_{j}^{F}$ (39), and this set contains $\hat{S}_{j}(35)$, that is,

$$
\hat{S}_{j}^{F} \supset \hat{S}_{j}
$$

The above fault scenario allows any sequence of persistent sensor faults, including simultaneous faults of several sensors, as long as the first fault occurs after sufficiently long time of operation without fault (such that all variables have entered the corresponding invariant sets) and at least one sensor remains operational.

The following theorem provides conditions to guarantee closed-loop stability under sensor fault.

Theorem 4.3 Suppose that bounds on the sensor noises $\eta_{i}$, on the "fault noises" $\eta_{i}^{F}$ and on the sensor reference signals $\xi_{j, r e f}$, for $i=1, \ldots, N$, are given in the form $\eta_{i} \in N_{i}, \eta_{i}^{F} \in N_{i}^{F}$ and $\xi_{i, r e f} \in \Xi_{i, r e f}$, respectively, where $N_{i}, N_{i}^{F}$ and $\Xi_{i, \text { ref }}$ are polyhedral sets. Also let $Z_{i}$ be an ultimate-bound set for the sensor tracking error dynamics (28). ${ }^{4}$ Suppose that the following conditions hold for all $j=1, \ldots, N$ :

$$
\max _{i}\left\{J_{i}^{\max }: i \in\{1, \ldots, N\}, i \neq j\right\}<J_{j}^{\min },
$$

where

$$
\begin{aligned}
& J_{i}^{\max } \triangleq \max \left\{\left(\hat{z}_{i}^{U P}\right)^{\prime} P \hat{z}_{i}^{U P}: \hat{z}_{i}^{U P}=T_{i} \theta_{i},\right. \\
& \left.\theta_{i} \in \hat{S}_{i} \times N_{i} \times Z_{i}\right\}, \\
& J_{j}^{m i n} \triangleq \min \left\{\left(\hat{z}_{j}^{U P}\right)^{\prime} P \hat{z}_{j}^{U P}: \hat{z}_{j}^{U P}=T_{j} \theta_{j}^{F},\right. \\
& \left.\theta_{j}^{F} \in \hat{S}_{j}^{F} \times N_{j}^{F} \times\left(-\Xi_{j, r e f}\right)\right\}, \\
& T_{i} \triangleq\left[I-M_{i} C_{s_{i}} M_{i} M_{i} C_{s_{i}}\right], \\
& \theta_{i} \triangleq\left[\begin{array}{llll}
\hat{z}_{i}^{\prime} & \hat{\zeta}_{i}^{\prime} & \eta_{i}{ }^{\prime} & \zeta_{i}^{\prime}
\end{array}\right]^{\prime}, \theta_{j}^{F} \triangleq\left[\begin{array}{llll}
\hat{z}_{j}^{\prime} & \hat{\zeta}_{j}^{\prime} & \eta_{j}^{F^{\prime}}-\xi_{j, r e f}^{\prime}
\end{array}\right]^{\prime} \text {. }
\end{aligned}
$$

Then, under the fault scenario of Assumption 4.2, the closed-loop dynamics of the multisensor switching scheme described in Section 2 remain stable in the event any sensor fails.

PROOF. For all healthy sensors, using (18), (19), (4), (15), (17), (44)-(45) we can write $\hat{z}_{i}^{U P}=\hat{z}_{i}+\gamma_{i}=T_{i} \theta_{i}$. On the other hand, using (18), (19), (36), (17), (44)(45), we can write for the faulty sensor $\hat{z}_{j}^{U P}=\hat{z}_{j}+\gamma_{j}=$

4 The set $Z_{i}$ can be computed using ultimate bounds on $z$ and applying Theorem 3.2 to (28). 
$T_{j} \theta_{j}^{F}$. Thus, using (25), conditions (41)-(43) ensure that the largest possible value of the switching cost over all healthy sensors whose estimator tracking error states belong to $\hat{S}_{i}(35)$, is smaller than the smallest possible cost value achievable by the faulty $j$ th sensor, whenever the latter has estimator tracking error states in $\hat{S}_{j}^{F}(39)$.

Suppose now that a $j$ th sensor fails. At the time of the fault, Condition 1-(b) of Assumption 4.2 guarantees that there exists at least one operational $l$ th sensor that has the states of the corresponding estimator tracking error subsystem (34) in the invariant set $\hat{S}_{l}$. In addition, by Condition 2 of Assumption 4.2, the states of the estimator tracking error subsystem corresponding to the failed sensor are in $\hat{S}_{j}^{F}$. Conditions (41)-(43) then ensure that the $l$ th sensor has smaller cost than the failed $j$ th sensor and thus the latter cannot be selected by the switching controller. It follows that at the time of the fault the controller selects any of the available healthy sensors (not necessarily the $l$ th sensor) which, by Condition 1-(b) of Assumption 4.2 has estimation errors inside $\tilde{S}_{i}(31)$, hence satisfying the bounds (32). Thus Assumption 4.1 holds at the time of the fault and the analysis of Section 4.1 shows that the states of the estimator tracking error subsystems corresponding to healthy sensors and to the failed $j$ th sensor remain in $\hat{S}_{i}$ and $\hat{S}_{j}^{F}$, respectively. The previous argument can be repeated inductively for the duration of the fault, concluding that the switching controller never selects faulty sensors to implement the control law and that the resulting dynamics remain in the respective invariant sets. The result then follows.

\section{Implementation issues}

The on-line implementation of the switching controller involves simple comparisons needed to evaluate (24). The fault tolerance guarantees of the scheme, on the other hand, can be tested off line. Indeed, for a given set of system parameters (such as bounds on the sensor noises, maximum and minimum values of the reference signal, etc.), conditions (40) and (41)-(43) can be checked before the implementation of the scheme. To check condition (40), note that it holds if and only if

$$
\left(I-\left|\Lambda_{j}\right|\right)^{-1}\left|V_{j}^{-1}\left[\begin{array}{cc}
L_{j} C_{s_{j}} & L_{j} \\
L_{s_{j}} C_{s_{j}} & L_{s_{j}}
\end{array}\right]\right|\left[\begin{array}{c}
\bar{\xi}_{j, r e f}-\bar{\zeta}_{j} \\
\bar{\eta}_{j}^{F}-\bar{\eta}_{j}
\end{array}\right]>0
$$

where the 'bars' denote bounds on the elementwise magnitude of the corresponding signal [see (35), (39) and the definitions (33) and (37)]. Thus (40) can be satisfied by appropriate selection of bounds on $\left|\xi_{j, r e f}\right|$ and $\left|\eta_{j}^{F}\right|$. The evaluation of conditions (41)-(43) is particularly simple if the sets $Z_{i}$ are polyhedral (see footnote 4 ) and the eigenvalues of the matrices $A_{L_{j}}$ are real (which implies that the sets (35) and (39) are polyhedral, see Remark 3.4). In this case, the maximum in (42) is achieved at one of the vertices of the set $\hat{S}_{i} \times N_{i} \times Z_{i}$ and the minimisation in (43) is a (semidefinite) quadratic programme. Thus, the optimisations required by the off-line tests can be easily solved using standard numerical algorithms (see also Section 6 below).

\section{Geometric interpretation}

In this section we give a geometric interpretation of conditions (41)-(43). We require the following definitions. If $X \subset \mathbb{R}^{n}$ and $Y \subset \mathbb{R}^{n}$ are some sets, their sum is the set $X+Y=\left\{z \in \mathbb{R}^{n}: z=x+y, x \in X, y \in Y\right\}$. If $T \in$ $\mathbb{R}^{n \times m}$ is a matrix and $X \subset \mathbb{R}^{m}$ is a set, the set $T X \subset \mathbb{R}^{n}$ is defined as $T X=\left\{z \in \mathbb{R}^{n}: z=T x, x \in X\right\}$.

Next, introduce the variable $s_{i} \triangleq P^{1 / 2} \hat{z}_{i}^{U P}$, where $P^{1 / 2}$ is the symmetric square root of the switching cost matrix $P$. Using the definitions (44)-(45) and the sets

$$
\begin{aligned}
& S_{i}^{\text {max }} \triangleq P^{1 / 2}\left\{\left[I-M_{i} C_{s_{i}}\right] \hat{S}_{i}+M_{i} N_{i}+M_{i} C_{s_{i}} Z_{i},\right\}, \\
& S_{j}^{\text {min }} \triangleq P^{1 / 2}\left\{\left[I-M_{j} C_{s_{j}}\right] \hat{S}_{j}^{F}+M_{j} N_{j}^{F}-M_{j} C_{s_{j}} \Xi_{j, r e f}\right\},
\end{aligned}
$$

we have that (42) and (43) have the equivalent form

$$
\begin{aligned}
J_{i}^{\max } & =\max \left\{\left\|s_{i}\right\|^{2}: s_{i} \in S_{i}^{\max }\right\}, \\
J_{j}^{\min } & =\min \left\{\left\|s_{j}\right\|^{2}: s_{j} \in S_{j}^{\text {min }}\right\},
\end{aligned}
$$

where $\|\cdot\|$ is the vector 2-norm. Thus, $J_{i}^{\max }$ is the $\max$ imum squared-norm over all vectors in $S_{i}^{\max }$ and $J_{j}^{\min }$ is the minimum squared-norm over all vectors in $S_{j}^{\text {min }}$. Clearly, for (41) to hold for a particular index $i$, the sets $S_{i}^{\text {max }}$ and $S_{j}^{\text {min }}$ must be separated by a sphere in $\mathbb{R}^{n}$ centred at zero. Figure 2 shows an illustration of a case where condition (41) holds for particular indices $i$ and $j$.

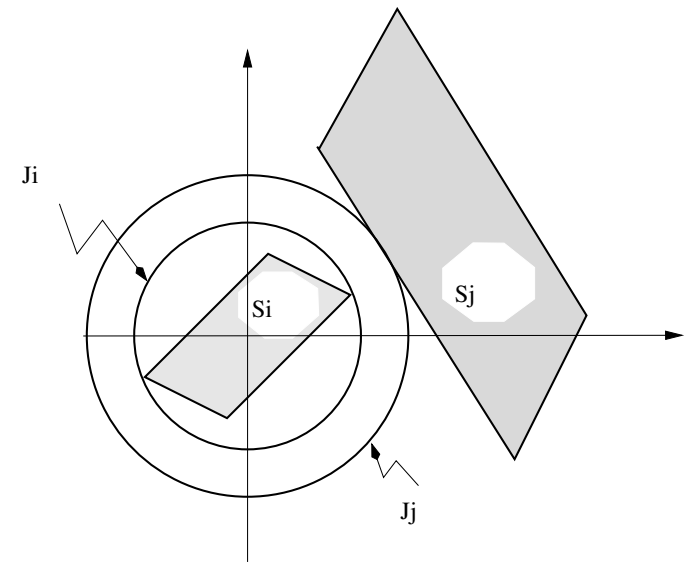

Fig. 2. Geometric interpretation of conditions (41)-(43). 
We next explain a mechanism by which the above separation can be achieved in practice. The sets $\hat{S}_{i}, Z_{i}$ and $\hat{S}_{j}^{F}$ are centred at zero since, in their computation, the process and (operational) measurement noise bounds are considered centred at zero (recall, for example, that in the computation of $\hat{S}_{j}^{F}$ in (39) we used bounds of the form $\left.\left|\nu_{l j}^{F}\right| \leq \bar{\nu}_{l j}^{F}\right)$. Hence, if $N_{i}$ is also centred at zero (which is typically the case), then the same is true for the set $S_{i}^{\text {max }}$ in (47), which is thus equal to the sum of three sets centred at zero. The set $S_{j}^{\min }$ in (48) is also the sum of three sets. The first set, $P^{1 / 2}\left[I-M_{j} C_{s_{j}}\right] \hat{S}_{j}^{F}$, is centred at zero as just discussed. Therefore, to achieve the desired separation, the "fault noise" sets $N_{j}^{F}$ and/or the reference related sets $\Xi_{j, r e f}$ must necessarily be centred away from zero. Depending on the application, the former can be achieved, for example, by utilising preliminary "built-in" fault detection mechanisms in the sensors (Hsiao and Tomizuka, 2005) while the latter can be achieved if the reference coordinate frame is off set away from zero. Note then that regulation problems around zero can only be handled by the scheme using preliminary fault detection mechanisms in the sensors.

Once the conditions for separation of the sets are fulfilled, then one could, in principle, perform fault detection and isolation using this information. Here we have chosen to achieve faulty sensor detection and isolation "implicitly" by guaranteeing that the switching cost avoids selecting faulty sensors. This feature of the proposed scheme, we believe, departs from other available techniques to achieve fault detection and isolation. Notice also that the proposed switching scheme is motivated by a control performance criterion and has a good performance in the absence of sensor failure. The possibility to provide fault tolerance guarantees with the scheme without any modification comes as a bonus. Moreover, a nice property of the proposed scheme is the simplicity of its on-line implementation, which requires only to compare cost values. On the other hand, the use of the separation of the sets as a mechanism for fault detection and isolation would result in a relatively more complex scheme requiring more involved on-line tests.

\section{Automotive vehicle longitudinal control}

We consider here the longitudinal control problem for a car following scenario. In this scenario, the follower car approaches the leader car at a high speed until the follower car velocity equals the leader's one and the interdistance between the two cars takes the desired value and remains constant thereafter (Martínez and Canudas de Wit, 2004). The automotive longitudinal control is generally composed by an inner control loop which compensates the nonlinear vehicle dynamics, and an outer control loop which is responsible for tracking of the desired interdistance reference.
This example is concerned with the outer interdistance control loop. The interdistance dynamics are represented by the discretisation of a double integrator plant, for a sample time of $0.1 \mathrm{~s}$, and satisfy (1) with $A=\left[\begin{array}{cc}1 & 0.1 \\ 0 & 1\end{array}\right], B=\left[\begin{array}{ll}0 & 0.1\end{array}\right]^{\prime}, E=B$ and $|w| \leq 0.02$. The two sensor dynamics are modelled by (3)-(4) with ${ }^{5}$ $C=\left[\begin{array}{ll}1 & 0\end{array}\right]$ and $A_{s_{1}}=0.6065, B_{s_{1}}=0.5, C_{s_{1}}=0.7869$, $A_{s_{2}}=0.8187, B_{s_{2}}=0.5, C_{s_{2}}=0.3625,\left|\eta_{i}\right| \leq 0.1$, $\left|\eta_{i}^{F}\right| \leq 1$ [cf. (36)], for $i=1,2$. The estimators are given by (6)-(9) with $L_{1}=[1.10640 .3010]^{\prime}, L_{s_{1}}=1.3089$, $L_{2}=\left[\begin{array}{ll}1.1945 & 0.2985\end{array}\right]^{\prime}, L_{s_{2}}=2.8405$ (computed by pole placement) and $\left[\begin{array}{c}M_{i} \\ M_{s_{i}}\end{array}\right]=\left[\begin{array}{cc}A & 0 \\ B_{s_{i}} C & A_{s_{i}}\end{array}\right]^{-1}\left[\begin{array}{c}L_{i} \\ L_{s_{i}}\end{array}\right]$, for $i=1,2$. The Jordan decompositions $A_{L_{i}}=V_{i} \Lambda_{i} V_{i}^{-1}$ of (10) are computed using Matlab's eig function. The controller is designed as in Section 2.3 with $Q=$ $\left[\begin{array}{cc}0.1007 & 0 \\ 0 & 6.3187\end{array}\right], R=7.2598, K=[0.11181 .0091]$ We assume that the leader car has a constant velocity of $20 \mathrm{~m} / \mathrm{s}$, the initial interdistance between leader and follower cars is $75 \mathrm{~m}$, and the initial velocity of the follower car is $30 \mathrm{~m} / \mathrm{s}$. Using the interdistance reference model of Martínez and Canudas de Wit (2004) with the above problem data gives an elementwise bounded reference tracking signal $[33-10]^{\prime} \leq x_{\text {ref }} \leq\left[\begin{array}{ll}75 & 0.08\end{array}\right]^{\prime}$.

With the above data, the set condition (40) [and equivalently (46)], for $i=1,2$, and conditions (41)-(43) are satisfied. Hence the system is guaranteed to be closedloop stable under sensor fault. The geometric conditions for (41)-(43), described in Section 6, are shown for this particular example in Figure 3.

In the simulation, each sensor fails for a period of $20 \mathrm{~s}$ and then recovers, with no fault periods overlapping between sensors (since we require at least one operational sensor at all times). Sensor 1 fails during $20 \leq t \leq 40$ and sensor 2 fails during $70 \leq t \leq 90$. Figure $4 a$ ) depicts the interdistance reference signal (dotted) and the actual interdistance between vehicles (solid). The switching sequence, shown in Figure 4b), commutes between both sensors in the absence of fault and chooses exclusively the healthy sensor during faults. The updated estimate of the interdistance between vehicles is shown in Figure 4c) for sensor 1's estimator, and in Figure 4d) for sensor 2's estimator. The shaded areas in Figures $4 \mathrm{~b}$ )4f) indicate the period when a sensor is failed. Notice that the switching system exhibits a stable behaviour for the fault scenario described in Assumption 4.2.

\footnotetext{
5 We simulate a slow sensor (stereo-vision), and a fast sensor (laser). They are both modelled as low pass filters, where the chosen bandwidths characterise the necessary time for signal processing (Gavrila et al., 2001).
} 

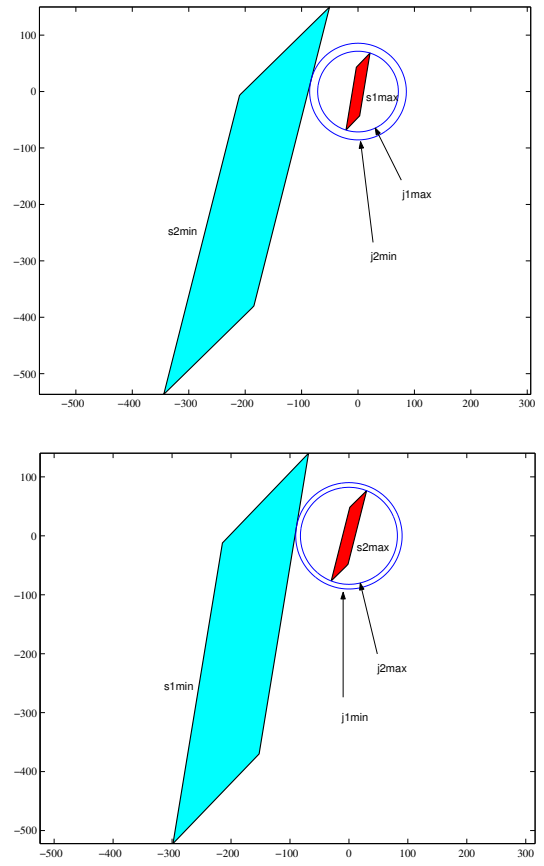

Fig. 3. Geometric interpretation of the multisensor switching stability conditions (41)-(43) for longitudinal control.
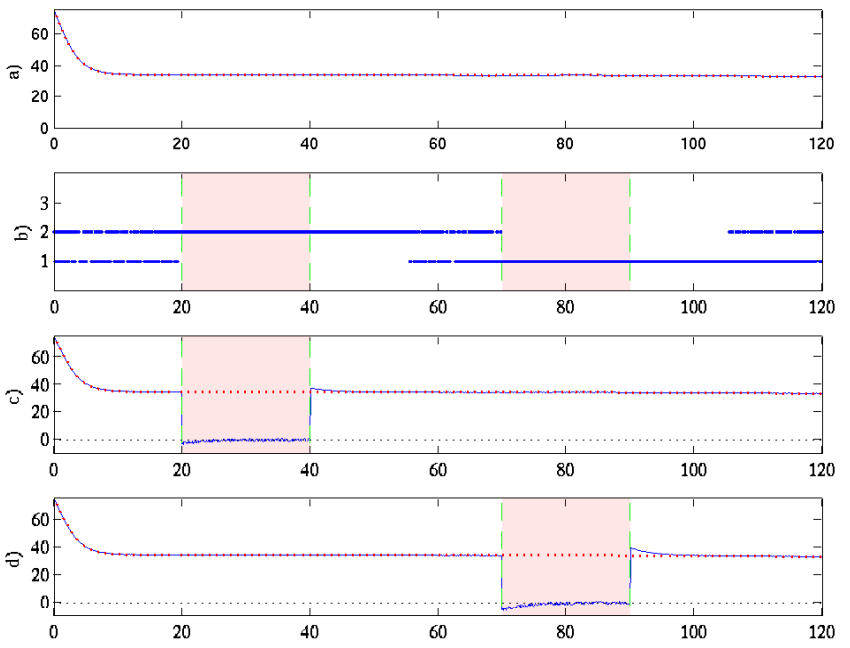

Fig. 4. Longitudinal vehicle control using the multisensor switching scheme. a) Interdistance reference signal (dotted) and actual interdistance (solid). b) Switching sequence. c), d) Interdistance reference (dotted) and updated estimate of the interdistance using sensor 1 and 2, respectively.

\section{Comparison with a fusion-based scheme}

In this section we compare the tracking performances between the multisensor switching control scheme of Section 2 and an ad-hoc fault tolerant control scheme, based on sensor fusion, proposed in Zhuo et al. (2006). We con- sider a system of the form (1) with $A=1, B=0.1$, $E=0.01$ and $|w| \leq 0.5$. The state $x$ is measured via a set of 3 identical sensors of the form (3)-(4) with $B_{s_{i}} C=0.5, A_{s_{i}}=0.6065, C_{s_{i}}=0.7869,\left|\eta_{i}\right| \leq 0.5$, and $\left|\eta_{i}^{F}\right| \leq 0.5$ [cf. (36)], for $i=1,2,3$. The reference signal $x_{r e f}$ satisfies (2) and is bounded as $4 \leq x_{r e f} \leq 6$.

\subsection{Fault tolerant multisensor switching scheme}

The estimators are given by (6)-(9) with $L_{i}=M_{i}=$ 0.9999, $L_{s_{i}}=M_{s_{i}}=1.2707$, for $i=1,2,3\left(L_{i}\right.$ and $L_{s_{i}}$ are computed by pole placement). The Jordan decompositions $A_{L_{i}}=V_{i} \Lambda_{i} V_{i}^{-1}$ of (10) are computed using Matlab's eig function. The controller is designed as in Section 2.3 with $Q=1, R=16$, yielding $K=0.2469$. The above data satisfy the invariant set condition (40) [and equivalently (46)], for $i=1,2,3$, and conditions (41)(43). Thus, Theorem 4.3 guarantees closed-loop stability of the ensuing feedback control system using the multisensor switching scheme described in Section 2.

\subsection{Fusion-based scheme}

In the scheme of Zhuo et al. (2006), each sensor has an associated Kalman filter (KF) of the form (6)-(9). For each KF, a fault detection and isolation (FDI) unit performs fault detection using hypothesis testing procedures (Mehra and Peschon, 1971). For all sensors considered healthy by the FDI unit, the corresponding updated tracking error estimates $\hat{z}_{i}^{U P}$ [cf. (18)] are combined to form the optimal (in the linear minimum variance sense) information fusion Kalman filter estimate $\hat{z}^{o}$ (Sun and Deng, 2004). The feedback control action is then computed using $\hat{z}^{o}$ with the feedback gain $K$ of Section 8.1. The disturbances $w, \eta_{i}$ and $\eta_{i}^{F}, i=1,2,3$, are i.i.d zero mean Gaussian white noise sequences with variance 0.0156 , independent from each other. The initialisation values for the KFs are $\hat{x}_{i}(0 \mid 0)=0, \hat{\xi}_{i}(0 \mid 0)=0$ and the error covariance $P_{i}(0 \mid 0)=0$ for $i=1,2,3$.

\subsection{Simulation comparison}

We perform 100 runs under identical conditions but with different noise realisations. To satisfy the assumptions required by both schemes, all 100 realisations of the Gaussian noises for the simulation are selected as explained in Section 8.2 and such that they are bounded as $|w| \leq 0.5,\left|\eta_{i}\right| \leq 0.5$, and $\left|\eta_{i}^{F}\right| \leq 0.5, i=1,2,3$. In all 100 runs, sensor 1 fails during $50 s \leq t \leq 100 \mathrm{~s}$ and sensors 2 and 3 fail during $150 s \leq t \leq 200 s$. Figure 5a) depicts the reference signal $x_{r e f}$ (dotted), and the average over all runs of the plant state responses using the switching scheme (solid) and using the fusion-based scheme (dashed). Figure 5b) plots the average tracking error $z$ using the multisensor switching scheme (solid) and the fusion-based scheme (dashed). Both schemes exhibit similar tracking performances during the periods 

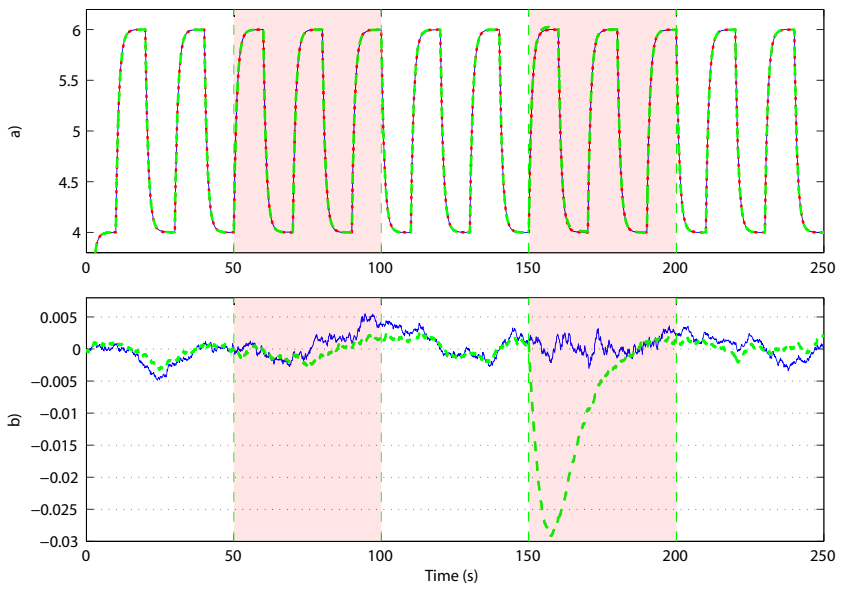

Fig. 5. Tracking comparison between the proposed switching scheme (solid) and the fusion-based scheme of Zhuo et al. (2006) (dashed).

when all sensors are healthy, with a slightly better performance for the fusion-based scheme. Thus, although the switching scheme might seem to discard useful information by selecting only one healthy sensor at each time, the performance is not degraded with respect to that of the fusion-based scheme. When two sensors fail the average performance of the fusion-based scheme is noticeably degraded. This is due to the fact that, especially during $150 s \leq t \leq 160 s$ the FDI unit gives false alarms in most runs, that is, it wrongly deems all sensors faulty and hence the scheme works in open loop. On the other hand, the switching mechanism maintains a similar performance during all simulation. We observe that the proposed switching scheme is relatively easy to implement (see Section 5), whereas the fusion-based scheme requires the execution of several tests in the FDI units. Moreover, there are not known guaranteed stability properties for the fusion-based scheme while in contrast, as proved in this paper, closed-loop stability can be guaranteed for the multisensor switching scheme under the fault scenario of Assumption 4.2.

\section{Conclusions}

We have proposed a new multisensor switching strategy for feedback control and have established closedloop stability under fault-free operation. In addition, we have shown that closed-loop stability is preserved under a multiple fault scenario if a set of conditions on the parameters of the problem is satisfied. We have discussed how an appropriate selection of the parameters (in particular, sensor-under-fault characteristics and reference signals) can be made to satisfy the required conditions. When compared with other fault tolerant multisensor schemes, our strategy has the important advantage that robust closed-loop stability under sensor fault can be guaranteed a priori, provided the aforementioned conditions are satisfied. We have applied the strategy to an example of automotive longitudinal control.

\section{References}

M.S. Branicky. Multiple Lyapunov functions and other analysis tools for switched and hybrid systems. IEEE Trans. on Automatic Control, 43(4):475-482, 1998.

B.V. Dasarathy. Sensor fusion potential exploitationInnovative architectures and illustrative applications. Proceedings of the IEEE, 85(1):24-38, January 1997.

D.M. Gavrila, M. Kunert, and U. Lages. A multi-sensor approach for the protection of vulnerable traffic participants - the PROTECTOR project. In Proc. of the IEEE Inst. and Meas. Technology Conference, volume 3, pages 2044-2048, Budapest, Hungary, 2001.

T. Hsiao and M. Tomizuka. Sensor fault detection in vehicle lateral control systems via switching Kalman filtering. In Proc. of the 2005 American Control Conference, pages 5009-5014, Portland, OR, USA, 2005.

M.K. Kalandros, L. Trailović, L.Y. Pao, and Y. BarShalom. Tutorial on multisensor management and fusion algorithms for target tracking. In Proc. of the 2004 American Control Conference, volume 5, pages 4734-4748, Boston, MA, USA, June 2004.

E.J. Kofman, H. Haimovich, and M.M. Seron. A systematic method to obtain ultimate bounds for perturbed systems. International Journal of Control, 80(2):167178, February 2007.

R.C. Luo and M.G. Kay. Multisensor integration and fusion in intelligent systems. IEEE Trans. on Systems, Man and Cybernetics, 19(5):901-931, Sept.-Oct. 1989.

R.C. Luo, C.-C. Yih, and K.L. Su. Multisensor fusion and integration: approaches, applications, and future research directions. IEEE Sensors Journal, 2(2):107119, April 2002.

J.J. Martínez and C. Canudas de Wit. Model reference control approach for safe longitudinal control. In Proc. of the 2004 American Control Conference, Boston, MA, USA, June 2004.

R.K. Mehra and J. Peschon. An innovations approach to fault detection and diagnosis in dynamic systems. Automatica, 7:637-640, 1971.

M.M. Seron, X.W. Zhuo, J.A. De Doná, and J.J. Martínez. Fault tolerant multisensor switching control strategy. Technical report, School of Elec. Eng. and Computer Sci., The University of Newcastle, 2006. www.ee.newcastle.edu.au/reports/EE06014.pdf.

S.-L. Sun and Z.-L. Deng. Multi-sensor optimal information fusion Kalman filter. Automatica, 40(6):10171023, June 2004.

P.K. Varshney. Multisensor data fusion. Electronics \& Communication Eng. Journal, 9(6):245-253, 1997.

L. Xu, J.Q. Zhang, and Y. Yan. A wavelet-based multisensor data fusion algorithm. IEEE Trans. on Instrumentation and Measurement, 53(6):1539-1545, 2004.

X.W. Zhuo, M.M. Seron, and J.A. De Doná. Sensor fusion control with fault detection and isolation via hypothesis testing. Technical report, School of Elec. Eng. and Computer Sci., The University of Newcastle, 2006. www.ee.newcastle.edu.au/reports/EE06015.pdf. 